\title{
GENERALIZATION OF CHIRAL SYMMETRY FOR TILTED DIRAC CONES
}

\author{
TOHRU KAWARABAYASHI \\ Department of Physics, Toho University, Miyama 2-2-1 \\ Funabashi, 274-8510, Japan \\ tkawa@ph.sci.toho-u.ac.jp \\ YASUHIRO HATSUGAI \\ Institute of Physics, University of Tsukuba, \\ Tsukuba, 305-8571 Japan \\ TAKAHIRO MORIMOTO and HIDEO AOKI \\ Department of Physics, University of Tokyo, \\ Hongo, Tokyo, 113-0033 Japan
}

\begin{abstract}
The notion of chiral symmetry for the conventional Dirac cone is generalized to include the tilted Dirac cones, where the generalized chiral operator turns out to be nonhermitian. It is shown that the generalized chiral symmetry generically protects the zero modes $(n=0$ Landau level) of the Dirac cone even when tilted. The present generalized symmetry is equivalent to the condition that the Dirac Hamiltonian is elliptic as a differential operator, which provides an explicit relevance to the index theorem.
\end{abstract}

Keywords: massless Dirac fermions; chiral symmetry; zero modes.

PACS numbers: 73.43.-f, 11.30.Rd, 73.61.Ph

\section{Introduction}

The chiral symmetry plays an important role in specifying some of the universality classes of the critical phenomena in disordered systems. ${ }^{1-3}$ Systems are called chiral-symmetric when there exists an operator $\Gamma$ that anti-commutes with the Hamiltonian, $\{\Gamma, H\}=0$, with $\Gamma^{\dagger}=\Gamma$ and $\Gamma^{2}=1$. With this symmetry the energy eigenvalues appear always in pairs $(E,-E)$, since if we have an eigenstate $\psi_{E}$ with an eigenvalue $E$, the state $\Gamma \psi_{E}$ is an eigenstate with an eigenvalue $-E$. The energy spectrum is therefore exactly particle-hole symmetric even when there exists a disorder as far as the disorder respects the chiral symmetry. In particular, the zero-energy state can be expressed as an eigenstate of $\Gamma$.

For a massless Dirac fermions in two dimensions, usually with vertical and isotropic Dirac cones as in graphene, ${ }^{4-6}$ the effective Hamiltonian is expressed as $H_{0}=v_{F}\left(\sigma_{x} \pi_{x}+\sigma_{y} \pi_{y}\right)$, where $\sigma_{x(y)}$ is the Pauli matrix and $\boldsymbol{\pi}=\boldsymbol{p}+e \boldsymbol{A}$ denotes the 
dynamical momentum with the vector potential $\boldsymbol{A}$ and the electron charge $-e$. The fermi velocity is denoted by $v_{F}$. For such a vertical Dirac cone, we have obviously $\left\{H_{0}, \sigma_{z}\right\}=0$ and thus the system is chiral-symmetric with $\Gamma=\sigma_{z}$. The zero-energy Landau level (zero modes), which is essential to the anomalous quantum Hall effect for massless Dirac fermions in a magnetic field, then becomes an eigenstate of $\sigma_{z}$. The most remarkable property with these zero modes is their robustness against disorder in gauge degrees of freedom. The zero energy $(n=0)$ Landau level does not acquire any width due to such a disorder, while other Landau levels are broadened as usual, and this gives rise to an unconventional criticality for the quantum Hall transition at the $n=0$ Landau level. ${ }^{7}$ This robustness of zero modes for a vertical Dirac cone has been discussed in terms of the index theorem ${ }^{8,9}$ or based on the explicit form of wave functions due to Aharonov and Casher. ${ }^{10,11}$ It has been also demonstrated numerically that the chiral symmetry is also crucial to this robustness of zero modes of vertical Dirac cones. ${ }^{12}$

Massless Dirac fermions in two dimensions appear not only in graphene but also in certain organic metals, where we encounter pairs of tilted Dirac cones. ${ }^{13-17}$ The existence of zero modes and the Landau level structure have been established also for tilted Dirac cones. However, the effect of disorder, in particular the robustness of zero modes and the role of symmetry, has not been explored until recently. ${ }^{18}$ In the present paper, with an explicit form of the eigenstates of the generalized chiral operator, we demonstrate how the chiral symmetry, which is broken for tilted cones, can be generalized to include tilted Dirac cones and clarify its relevance to the robustness of the zero modes of generic massless Dirac fermions. Relationships between the generalized chiral symmetry and the applicability of the index theorem is also elaborated.

\section{Generalization of Chiral Symmetry}

To illustrate how the chiral symmetry is generalized to tilted Dirac cones, let us consider a general form of the Hamiltonian, ${ }^{18}$

$$
H(\eta)=-\eta v_{F} \sigma_{0} \pi_{x}+v_{F}\left(\sigma_{x} \pi_{x}+\sigma_{y} \pi_{y}\right),
$$

for a two-dimensional massless Dirac fermion in a magnetic field, where the isotropic Dirac cone is tilted in the $x$ direction for $\eta \neq 0$ with $\sigma_{0}$ being the unit matrix. The dynamical momentum $\pi$ satisfies the commutation relation $\left[\pi_{x}, \pi_{y}\right]=-i e \hbar B$ with $\boldsymbol{B}=\operatorname{rot} \boldsymbol{A}$. The parameter $\eta$ determines the tilting of the Dirac cone. Note that for $\boldsymbol{A}=0$ an equienergy contour of the Dirac cone is elliptic as long as $|\eta|<1$ (while hyperbolic for $|\eta|>1$; see Fig.1(a)). The first term in the Hamiltonian destroys the chiral symmetry as $\sigma_{z} H(\eta) \sigma_{z}=-H(-\eta) \neq-H(\eta)$. We can, however, define a generalized chiral operator $\gamma$ as

$$
\gamma=\lambda_{\eta}^{-1}\left(\sigma_{z}-i \eta \sigma_{y}\right), \quad \lambda_{\eta}=\sqrt{1-\eta^{2}}
$$

which arises naturally in the general framework of the eigenvalue problem for tilted Dirac cones. ${ }^{19}$ Although the generalized chiral operator $\gamma$ is not hermitian $\left(\gamma^{\dagger} \neq \gamma\right)$, 
one can readily verify that $\gamma^{2}=1$ with eigenvalues \pm 1 . The corresponding righteigenvectors $\gamma| \pm\rangle= \pm| \pm\rangle$ are given explicitly as

$$
|+\rangle=\frac{1}{\sqrt{2\left(1+\lambda_{\eta}\right)}}\left(\begin{array}{c}
1+\lambda_{\eta} \\
\eta
\end{array}\right), \quad|-\rangle=\frac{1}{\sqrt{2\left(1+\lambda_{\eta}\right)}}\left(\begin{array}{c}
\eta \\
1+\lambda_{\eta}
\end{array}\right) .
$$

In the limit of the vertical cone $(\eta \rightarrow 0)$, the generalized operator $\gamma$ reduces to the conventional chiral operator $\sigma_{z}$. With the generalized chiral operator, we find that for $|\eta|<1$,

$$
\gamma^{\dagger} H(\eta) \gamma=-H(\eta)
$$

which we call the generalized chiral symmetry. ${ }^{18}$ The generalized symmetry guarantees the identity $\langle+|H(\eta)|+\rangle=\langle-|H(\eta)|-\rangle=0$, and plays an essential role for the robustness of zero modes as shown in the next section. Note that this symmetry holds irrespective of the details of the vector potential $\boldsymbol{A}$. Disorder in gauge degrees of freedom (such as random magnetic fields) respects this symmetry.

\section{Robustness of Zero Modes}

If the above generalized chiral symmetry is preserved, the Schrödinger equation $H(\eta) \psi=E \psi$ for the wave function $\psi=|+\rangle \psi^{+}+|-\rangle \psi^{-}$becomes

$$
\left(\begin{array}{cc}
0 & \lambda_{\eta} \pi_{x}-i \pi_{y} \\
\lambda_{\eta} \pi_{x}+i \pi_{y} & 0
\end{array}\right)\left(\begin{array}{c}
\psi^{+} \\
\psi^{-}
\end{array}\right)=E\left(\begin{array}{cc}
1 / \lambda_{\eta} & \eta / \lambda_{\eta} \\
\eta / \lambda_{\eta} & 1 / \lambda_{\eta}
\end{array}\right)\left(\begin{array}{c}
\psi^{+} \\
\psi^{-}
\end{array}\right) .
$$

The zero $(E=0)$ modes are then given by the wave functions satisfying ${ }^{18}$

$$
\left(\lambda_{\eta} \pi_{x}-i \pi_{y}\right) \psi^{-}=0, \psi^{+}=0
$$

or

$$
\left(\lambda_{\eta} \pi_{x}+i \pi_{y}\right) \psi^{+}=0, \psi^{-}=0 .
$$

The zero modes are thus the eigenstates of the generalized chiral operator $\gamma$ and have either "-" chirality with $\gamma \psi=-\psi$ (Eq.(1)) or "+" chirality with $\gamma \psi=\psi$ (Eq.(2)). It is to be recalled that for a vertical Dirac cone the zero modes are also the eigenstates of the chiral operator $\Gamma=\sigma_{z}$. These equations for the zero modes hold even in the case where the gauge field is disordered.

Following Aharonov and Casher, ${ }^{10}$ we adopt the "Coulomb gauge" $\lambda_{\eta} \partial_{x} A_{x}+$ $\lambda_{\eta}^{-1} \partial_{y} A_{y}=0$ by assuming $\boldsymbol{A}=\left(-\lambda_{\eta}^{-1} \partial_{y} \varphi, \lambda_{\eta} \partial_{x} \varphi\right)$. Then Eq.(1) for $\psi^{-}$and Eq.(2) for $\psi^{+}$are reduced to

$$
\left[D_{ \pm} \mp(e / \hbar)\left(D_{ \pm} \varphi\right)\right] \psi^{ \pm}=0
$$

where $D_{ \pm} \equiv\left(\partial_{X} \pm i \partial_{Y}\right)$ with $\boldsymbol{R}=(X, Y)=\left(x / \sqrt{\lambda_{\eta}}, y \sqrt{\lambda_{\eta}}\right)$. The solutios are then given by $\psi^{ \pm}=\exp ( \pm e \varphi / \hbar) f\left(Z_{ \pm}\right)$with a polynomial $f\left(Z_{ \pm}\right)$in $Z_{ \pm} \equiv$ $X \pm i Y$. Since $\left(\partial_{X}^{2}+\partial_{Y}^{2}\right) \varphi=B$, we have $\varphi(\boldsymbol{R})=\int d \boldsymbol{R}^{\prime} G\left(\boldsymbol{R}-\boldsymbol{R}^{\prime}\right) B\left(\boldsymbol{R}^{\prime}\right)$ where $G(\boldsymbol{R})=(1 / 2 \pi) \log (R)$ with $R=\sqrt{X^{2}+Y^{2}}$, which leads to the asymptotic form $\varphi \rightarrow(\Phi / 2 \pi) \log R$ in the limit as $R \rightarrow \infty$ with $\Phi$ being the total magnetic flux in the 
system. We then have to chose the chirality for the wave function to be normalizable. For instance, when the total magnetic flux $\Phi$ is positive, the zero mode has to have "-" chirality to be normalizable. The number of square-normalizable wave function then becomes $\Phi /(h / e),{ }^{10}$ that exactly coincides with the degeneracy of the Landau level. This implies the zero-modes exhaust the Landau level of a tilted Dirac cone so that its density of states is a generalized chiral symmetry protected delta-function in the presence of disorder. In this sense, the generalized chiral symmetry protects the zero mode of a generic massless Dirac fermions in two dimensions.

\section{Generalized Chiral Symmetry and Index Theorem}

The above reasoning gives an explicit relationship between the index theorem and the generalized chiral symmetry. The index theorem, which gives the least number of zero modes, holds for an elliptic operator. ${ }^{8}$ In the present case, the Hamiltonian $H(\eta)$ is elliptic as a differential operator if the matrix,

$$
\Xi\left(\xi_{x}, \xi_{y}\right)=\left(\begin{array}{cc}
-\eta \xi_{x} & \xi_{x}-i \xi_{y} \\
\xi_{x}+i \xi_{y} & -\eta \xi_{x}
\end{array}\right),
$$

is invertible for any $\left(\xi_{x}, \xi_{y}\right) \in \mathbf{R}^{2}-(0,0)$. The condition for the ellipticity thus becomes det $\Xi=-\left(1-\eta^{2}\right) \xi_{x}^{2}-\xi_{y}^{2} \neq 0$. This determinant is always negative (det $\Xi<$ 0 ) and never becomes zero for $\left(\xi_{x}, \xi_{y}\right) \in \mathbf{R}^{\mathbf{2}}-(0,0)$, as long as $|\eta|<1$. The condition $|\eta|<1$ is therefore exactly the condition for the ellipticity of the Hamiltonian $H(\eta)$ as well as that for the existence of the generalized chiral symmetry. In this sense, the generalized chiral symmetry is generically equivalent to the ellipticity of the Diraccone Hamiltonian with the same parameter space for their validity. Geometrically, the condition $|\eta|<1$ means that the Dirac cone is not tilted too much so that the cross section with a constant energy plane remains to be elliptic.

\section{Numerical Demonstration}

We have also preformed numerical calculations based on a lattice model having a pair of tilted Dirac cones at $E=0$ as shown in Fig.1(b)(Inset). The model is defined on the two-dimensional square lattice with a Hamiltonian having the nearest-neighbor $(t)$ and the next-nearest neighbor $\left(t^{\prime}\right)$ transfer integrals as ${ }^{18,20}$

$$
H=\sum_{\boldsymbol{r}}-t c_{\boldsymbol{r}+\boldsymbol{e}_{x}}^{\dagger} c_{\boldsymbol{r}}+(-1)^{x+y} t c_{\boldsymbol{r}+\boldsymbol{e}_{y}}^{\dagger} c_{\boldsymbol{r}}+t^{\prime}\left(c_{\boldsymbol{r}+\boldsymbol{e}_{x}+\boldsymbol{e}_{y}}^{\dagger} c_{\boldsymbol{r}}+c_{\boldsymbol{r}+\boldsymbol{e}_{x}-\boldsymbol{e}_{y}}^{\dagger} c_{\boldsymbol{r}}\right)+\text { H.c. },
$$

where $\boldsymbol{r}=(x, y)$ denotes the lattice point in units of the nearest-neighbor distance $a$, and $\boldsymbol{e}_{x}\left(\boldsymbol{e}_{y}\right)$ the unit vector in the $x(y)$ direction. The magnetic field is taken into account by the Peierls phases $\theta(\boldsymbol{r})$ as $t\left(t^{\prime}\right) \rightarrow t\left(t^{\prime}\right) \exp (-2 \pi i \theta(\boldsymbol{r}))$, so that the their sum along the closed loop is equal to the enclosed magnetic flux in units of the flux quantum $h / e$. To see how the Landau levels are broadened by disorder in the present model, we assume that the magnetic flux $\phi(r)$ enclosed by the square located at $\boldsymbol{r}$ has a random component $\delta \phi(\boldsymbol{r})$ in addition to the uniform component 
(a)
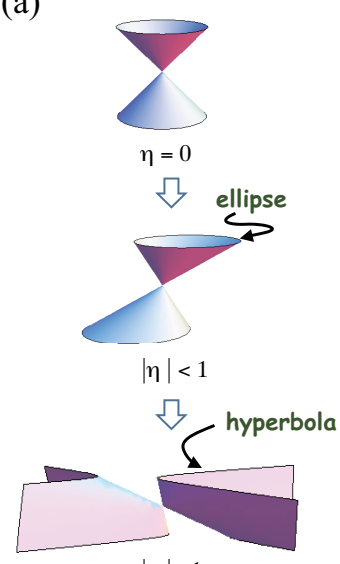

$|\eta|>1$ (b)

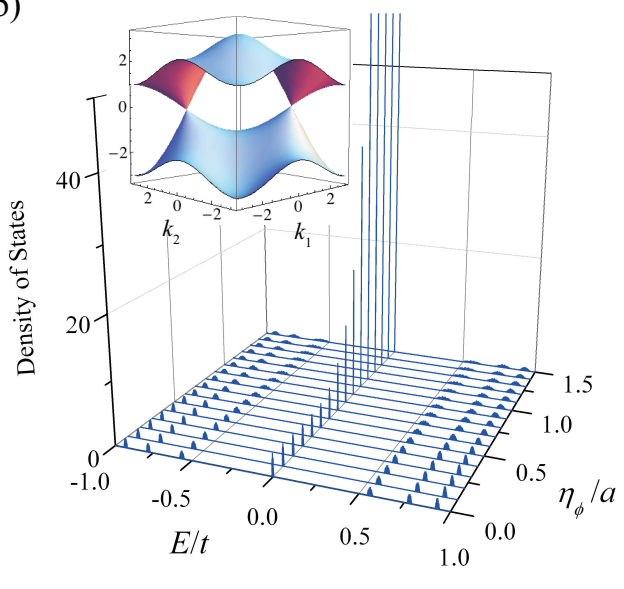

Fig. 1. (a) Schematic figures of the Dirac cones with $\eta=0,|\eta|<1$ and $|\eta|>1$. (b) Density of states for the lattice model in a magnetic field with various values of the correlation length $\eta_{\phi}$ of the random component of the magnetic flux. Here, the parameters are assumed to be $t^{\prime} / t=0.25$, $\phi=0.01(h / e)$, and $\sqrt{\left\langle\delta \phi^{2}\right\rangle}=0.0029(h / e)$. The system-size is $20 a$ by $20 a$ and the average over $10^{4}$ samples is made. Inset: Energy dispersion $E(\boldsymbol{k}) / t$ in the absence of a magnetic field, where $k_{1}=\boldsymbol{k} \cdot\left(\boldsymbol{e}_{x}+\boldsymbol{e}_{y}\right)$ and $k_{2}=\boldsymbol{k} \cdot\left(\boldsymbol{e}_{x}-\boldsymbol{e}_{y}\right)$.

$\phi$. The random components have a gaussian distribution and are correlated in space as $\left\langle\delta \phi\left(\boldsymbol{r}_{1}\right) \delta \phi\left(\boldsymbol{r}_{2}\right)\right\rangle=\left\langle\delta \phi^{2}\right\rangle \exp \left(-\left|\boldsymbol{r}_{1}-\boldsymbol{r}_{2}\right|^{2} / 4 \eta_{\phi}^{2}\right)$. The disorder in magnetic fluxes should appear, for large $\eta_{\phi}$, as the disorder in gauge degrees of freedom, and therefore should respect the generalized chiral symmetry of the effective Hamiltonian at the Dirac points. As shown in Fig.1(b), we actually see an anomalously sharp $n=0$ Landau level when the correlation length $\eta_{\phi}$ of disorder becomes larger than the nearest-neighbor distance $a$, while other Landau levels $(n= \pm 1, \pm 2, \pm 3)$ are broadened as usual. Together with the result ${ }^{18}$ for a different inclination of the cone, this anomaly at the $n=0$ Landau level is likely to exist universally, irrespective of the magnitude of the tilting angle of the cone. This anomalously sharp $n=0$ Landau level suggests that the energy levels are exactly degenerated at $E=0$. The present numerical results are thus consistent with the robustness of zero modes of a single tilted Dirac cone protected by the generalized chiral symmetry.

\section{Conclusions}

We have shown explicitly that the notion of the chiral symmetry can be generalized to generic tilted Dirac cones, where the generalized chiral operator has to be nonhermitian. It has been also demonstrated analytically that the generalized chiral symmetry indeed protects the zero modes of the system by extending the argument by Aharonov and Casher. ${ }^{10}$ The resulting anomalously sharp $n=0$ Landau level 
has been also confirmed numerically based on the lattice model. The existence of the generalized chiral symmetry coincides with the ellipticity of the Hamiltonian as a differential operator, which is nothing but the geometrical condition that the Dirac cone is not tilted too much so that the cross section with a constant energy plane is an ellipse.

\section{Acknowledgments}

The authors wish to thank Yoshiyuki Ono and Tomi Ohtsuki for useful discussions. This work was partly supported by Grants-in-Aid for Scientific Research, Nos. 22540336 and 23340112 from JSPS.

\section{References}

1. A.W.W. Ludwig, et al., Phys. Rev. B 50, 7526 (1994).

2. A. Altland and M.R. Zirnbauer, Phys.Rev. B 55, 1142 (1997).

3. F. Evers and A.D. Mirlin, Rev. Mod. Phys. 80, 1355 (2008).

4. K.S. Novoselov et al, Nature 438, 197 (2005).

5. Y. Zhang et al., Nature 438, 201 (2005).

6. A.H. Castro Neto et al, Rev. Mod. Phys. 81, 109 (2009).

7. P.M. Ostrovsky, I.V. Gornyi, and A.D. Mirlin, Phys. Rev. B 77, 195430 (2008).

8. M. Nakahara, Geometry, Topology, and Physics, 2nd ed. (Taylor \& Francis, 2003).

9. M.I. Katsnelson and K.S. Novoselov, Solid State Commun. 143, 3 (2007).

10. Y. Aharonov and A. Casher, Phys. Rev. A 19, 2461 (1979).

11. J. Kailasvuori, Europhys. Lett. 87, 47008 (2009).

12. T. Kawarabayashi, Y. Hatsugai, and H. Aoki, Phys. Rev. Lett. 103, 156804 (2009); Physica E42, 759 (2010); T. Kawarabayashi, T. Morimoto, Y. Hatsugai, and H. Aoki, Phys. Rev. B 82, 195426 (2010).

13. S. Katayama, A. Kobayashi, and Y. Suzuura, J. Phys. Soc. Jpn. 75, 054705 (2006).

14. N. Tajima, S. Sugawara, M. Tamura, Y. Nishio, and K. Kajita, J. Phys. Soc. Jpn. 75, 051010 (2006).

15. T. Morinari, T. Himura and T. Tohyama, J. Phys. Soc. Jpn. 78, 023704 (2009).

16. T. Morinari and T. Tohyama, J. Phys. Soc. Jpn. 79, 044708 (2010).

17. M.O. Goerbig, J.-N. Fuchs, G. Montambaux, and F. Piéchon, Phys. Rev. B 78, 045415 (2008).

18. T. Kawarabayashi, Y. Hatsugai, T. Morimoto, and H. Aoki, Phys. Rev. B 83, 153414 (2011).

19. Y. Hatsugai, T. Kawarabayashi, and H. Aoki, in preparation.

20. Y. Morita and Y. Hatsugai, Phys. Rev. Lett. 79, 3728 (1997); Y. Hatsugai, X.-G. Wen, and M. Kohmoto, Phys. Rev. B 56, 1061 (1997). 